Europhys. Lett., 9 (2), pp. 125-131 (1989)

\title{
Importance of Giant-Resonance Excitation for the Surface Properties of the Heavy-Ion Optical Potential.
}

\author{
Ph. Chomaz (*), Y. Blumenfeld $(* *)$, M. V. Andrés $(* * *)$ \\ F. Catara $(* *)\left(*_{*}^{*}\right)$ and E. G. Lanza $\left(*^{*} *\right)$ \\ $\left(^{*}\right)$ Division de Physique Théorique $\left(^{\S}\right)$, Institut de Physique Nucléaire \\ F-91406 Orsay Cedex, France \\ (**) Division de Recherche Expérimentale, Institut de Physique Nucléaire \\ F-91406 Orsay Cedex, France \\ (***) Departamento de Física Atómica y Nuclear, Universidad de Sevilla \\ 41080 Sevilla, Spain \\ $\left({ }^{*}{ }^{*}\right)$ Istituto di Fisica dell'Università - 98100 Messina, Italy \\ $\left(*_{*}^{*}\right)$ I.N.F.N., Sezione di Catania - 95129 Catania, Italy
}

(received 18 July 1988; accepted in final form 27 February 1989)

PACS. 25.70G - Compound nucleus properties and decays.

PACS. 25.70 - Heavy ion induced reactions and scattering.

\begin{abstract}
An imaginary potential associated with the inelastic excitation of the nuclei is computed in a multiphonon excitation model based on the random phase approximation. The surface properties of this potential are found to be dominated by giant-resonance degrees of freedom.
\end{abstract}

Recent studies of heavy-ion inelastic scattering have shown a strong excitation of both low-lying and giant vibrational states [1] and it appears now that heavy ions are one of the best probes for the investigation of these collective states [2]. Theoretical calculations [3, 4] have shown that multiple excitations of giant resonances may have detectable cross-sections $(\mathrm{d} \sigma / \mathrm{d} E \mathrm{~d} \Omega$ of the order of $1 \mathrm{mb} / \mathrm{sr} \cdot \mathrm{MeV}$ for the two-phonon state) and that these multiphonon states could be responsible for part of the high-energy strength observed in some reactions [5]. In the light of these developments it is important to assess the influence of such collective states on the global properties of heavy-ion reactions such as optical potentials. Indeed the inelastic excitation is related to a loss of flux in the elastic channel and thus to an imaginary potential $([6,7]$ and see eq. (7)). It will be shown that this absorption is strong and thus must be an important contribution to the total imaginary potential. The more interesting feature is that, at high incident energy, the surface properties and long-

(8) Laboratoire associé au C.N.R.S. 
range component of this inelastic potential are dominated by giant-resonance excitation. This long-range inelastic imaginary potential is in qualitative agreement with the results of ref. [8] obtained in a quantum-mechanical approach.

In this letter we use the multiphonon model presented in ref. [3, 4]. In this model the two nuclei follow a classical trajectory $R(t)$ which is obtained by solving the equations of motion with an ion-ion potential obtained by folding the target ground-state Hartree-Fock density with the projectile mean field and renormalizing by a factor 0.5 according to the prescription of Satchler [9] in order to simulate the effects of the Pauli principle between nucleons of the two nuclei [4]. For the Coulomb part a point charge potential was used. The energy and angular-momentum losses due to the inelastic excitation of the partners were not taken into account in the trajectory calculations. The excitations of the two nuclei are described microscopically within the random phase approximation (RPA). It must be noted that the use of RPA form factors can lead to appreciable differences with other similar calculations

TABLE I. - RPA response functions for the three nuclei considered in the calculations.

\begin{tabular}{|c|c|c|c|}
\hline Nucleus & $J^{\pi}$ & $E(\mathrm{MeV})$ & \% EWSR \\
\hline${ }^{40} \mathrm{Ca}$ & $\begin{array}{l}0^{+} \\
2^{+} \\
3^{-} \\
3^{-} \\
3^{-} \\
4^{+} \\
4^{+}\end{array}$ & $\begin{array}{r}20.00 \\
17.88 \\
3.56 \\
9.77 \\
32.99 \\
17.56 \\
41.00\end{array}$ & $\begin{array}{l}- \\
98.9 \\
18.6 \\
13.1 \\
67.3 \\
48.5 \\
48.0\end{array}$ \\
\hline${ }^{90} \mathrm{Zr}$ & $\begin{array}{l}0^{+} \\
1^{-} \\
2^{+} \\
2^{+} \\
2^{+} \\
3^{-} \\
3^{-} \\
3^{-} \\
4^{+} \\
4^{+} \\
4^{+} \\
4^{+} \\
5^{-}\end{array}$ & $\begin{array}{r}18.53 \\
31.00 \\
5.36 \\
15.77 \\
46.50 \\
2.59 \\
7.86 \\
29.95 \\
5.82 \\
7.93 \\
14.80 \\
44.00 \\
1.75\end{array}$ & $\begin{array}{r}-\overline{-} \\
\overline{8} .9 \\
77.9 \\
0.1 \\
10.5 \\
23.9 \\
51.4 \\
3.6 \\
5.0 \\
17.8 \\
40.0 \\
0.6\end{array}$ \\
\hline${ }^{208} \mathrm{~Pb}$ & $\begin{array}{l}0^{+} \\
2^{+} \\
2^{+} \\
2^{+} \\
3^{-} \\
3^{-} \\
4^{+} \\
4^{+} \\
4^{+} \\
4^{+} \\
5^{-} \\
5^{-} \\
5^{-} \\
5^{-}\end{array}$ & $\begin{array}{r}14.63 \\
5.19 \\
12.74 \\
39.18 \\
3.68 \\
24.25 \\
5.69 \\
9.56 \\
15.24 \\
35.44 \\
4.56 \\
19.16 \\
26.00 \\
46.54\end{array}$ & $\begin{array}{r}-\overline{14.4} \\
60.8 \\
16.7 \\
22.0 \\
33.7 \\
6.8 \\
6.4 \\
13.2 \\
17.3 \\
3.3 \\
7.9 \\
15.5 \\
3.4\end{array}$ \\
\hline
\end{tabular}


using the phenomenological form factors [10]. These differences are discussed in ref. [11]. The wave function of each nucleus excited by the mean field of its partner is found to be a coherent state

$$
|\psi(t)\rangle=\exp \left[-i_{\alpha}(t)\right]\left[\prod_{i} \exp \left[A_{0 i}(t) b_{i}^{\dagger}-\text { c.c. }\right]\right]|0\rangle,
$$

where $|0\rangle$ is the RPA ground state and $b_{i}^{\dagger}$ the creation of the one-phonon RPA state $|i\rangle=b_{i}^{\dagger}|0\rangle\left(b_{i}|0\rangle=0\right)$. We have used the RPA results of ref. [12] and the main properties of the RPA excited states can be found in table I. In eq. (1) $\alpha$ is a global phase and $A_{0 i}$ is the amplitude given by

$$
A_{0 i}(t)=\hbar^{-1} \int_{-\infty}^{t} \exp \left[i \omega_{i 0} t^{\prime}\right]\left\langle i\left|V\left(R\left(t^{\prime}\right)\right)\right| 0\right\rangle \mathrm{d} t^{\prime},
$$

where $\hbar \omega_{i 0}$ is the excitation energy of the state $|i\rangle$ and $V\left(R\left(t^{\prime}\right)\right)$ is the Hartree-Fock mean field of the partner travelling on the classical trajectory $R\left(t^{\prime}\right)$.

The probability amplitude for the system to remain in its ground state reads

$$
A_{0}=\langle 0 \mid \phi(t)\rangle=\exp [-i \alpha(t)] \exp [-1 / 2 \mathcal{N}(t)],
$$

where

$$
\mathcal{N}(t)=\sum_{i} \bar{n}_{i}=\sum_{i}\left|A_{i 0}(t)\right|^{2}
$$

In eq. (4) we have introduced the mean number of excited $i$-phonons $\bar{n}_{i}=\left|A_{i 0}\right|^{2}$. It must be noted that the sum $\sum_{i}$ runs over both target and projectile excitations. In this letter we will only discuss the imaginary part of the optical potential. One can define a transmission coefficient

$$
|S|^{2}=\exp [-\mathscr{N}(+\infty)]=\left|A_{0}\right|^{2}
$$

which only takes into account the absorption due to the inelastic excitation of the two partners (transfer reactions, for example, are not included). Several transmission coefficients are presented in fig. 1 as a function of entrance channel angular momenta $l$ for the three reactions $\mathrm{Ca}+\mathrm{Ca}, \mathrm{Ca}+\mathrm{Zr}$ and $\mathrm{Ca}+\mathrm{Pb}$ at $33 \mathrm{MeV}$ per nucleon. Their main feature is a rapid fall-off around the grazing trajectory when one considers decreasing angular momenta $l$.

In a semi-classical calculation an imaginary optical potential $W$ produces an absorption amplitude

$$
A_{0}=\exp \left[-i / h \int_{-\infty}^{t} \mathrm{~d} t^{\prime} W\left(t^{\prime}\right)\right]
$$

where the integration is performed along the classical trajectory. By equating eqs. (6) and (3) one gets

$$
\int_{-\infty}^{t} W\left(t^{\prime}\right) \mathrm{d} t^{\prime}=\frac{1}{2} \mathcal{N}^{\gamma}(t)
$$




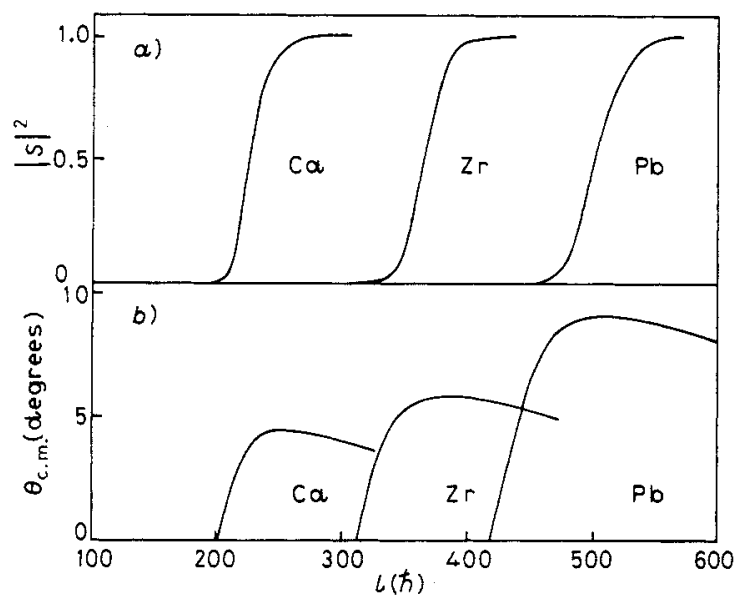

Fig. 1. - a) $|S|^{2}$ transmission coefficient (see eq. (5)) as a function of the entrance channel angularmomentum $l$ for the three studied reactions ${ }^{40} \mathrm{Ca}+{ }^{40} \mathrm{Ca},{ }^{40} \mathrm{Ca}+{ }^{90} \mathrm{Zr},{ }^{40} \mathrm{Ca}+{ }^{208} \mathrm{~Pb}$ at an incident energy of the calcium nucleus of $33 \mathrm{MeV}$ per nucleon. $b$ ) The three associated deflection functions.

Since we study the absorption arising from nuclear excitations we have restricted the potential $V$ in eq. (2) to be the nuclear potential. However, the Coulomb potential was included in the dynamical part of the calculation.

The validity of eq. (7) at any time $t$ is in fact too stringent a condition because the measurable quantities that can be extracted from this semi-classical approximation are the final amplitudes $A_{0}(t=+\infty)$. Moreover, it is shown in ref. [7] that eq. (7) yields $l$-dependent potentials which can be different in the entrance and exit channels (see also ref. [6]). Therefore, in order to define a local $l$-independent potential we have fitted the $|S|^{2}$ curve using the following procedure:

i) For each trajectory (i.e. each $l)|S(l)|^{2}$ is reproduced using several Woods-Saxon imaginary potentials with different radii and diffuseness. In fact, for a large set of values of the radius $(r)$ and diffuseness $(a)$ parameters a depth $W_{0}(r, a, l)$ is determined by applying eq. (7) at $t=+\infty$.

ii) Then the radius and diffuseness which yield a depth independent of $l$ (or which minimize the deviations of $W_{0}(l)$ from a constant value) are chosen. In the general case a small $l$-dependence of the potential remains (of the order of $10 \%$ in the region of interest) and thus this procedure defines a $l$-dependent imaginary potential $W_{l}(R)$.

iii) By using the fact that for each $l$ the turning point $(D(l))$ region gives the dominant contribution to the left-hand part of eq. (7), this residual dependence can be eliminated by retaining only the value of the Woods-Saxon at this point. We thus construct the $l$ independent imaginary potential $W$ by linking the values of the potentials obtained at the turning points of each trajectory $W(D(l))=W_{l}(D(l))$.

The geometry of this final potential is close to the Woods-Saxon geometry defined in ii) demonstrating the consistency of the method. In ref. [7] a slightly simplified procedure for the extraction of the $l$-independent potential was used. The good prediction found for elastic scattering data shows the validity of the general approach used here. The present paper focuses on the absorption mechanisms and thus particular care was taken to extract a potential fitting as closely as possible the $|S|^{2}$ curve.

The resulting potentials for the three reactions $\mathrm{Ca}+\mathrm{Ca}, \mathrm{Ca}+\mathrm{Zr}$ and $\mathrm{Ca}+\mathrm{Pb}$ at $33 \mathrm{MeV}$ per nucleon are presented in fig. 2a). Figure $2 b$ ) illustrates the evolution of $W$ with incident 

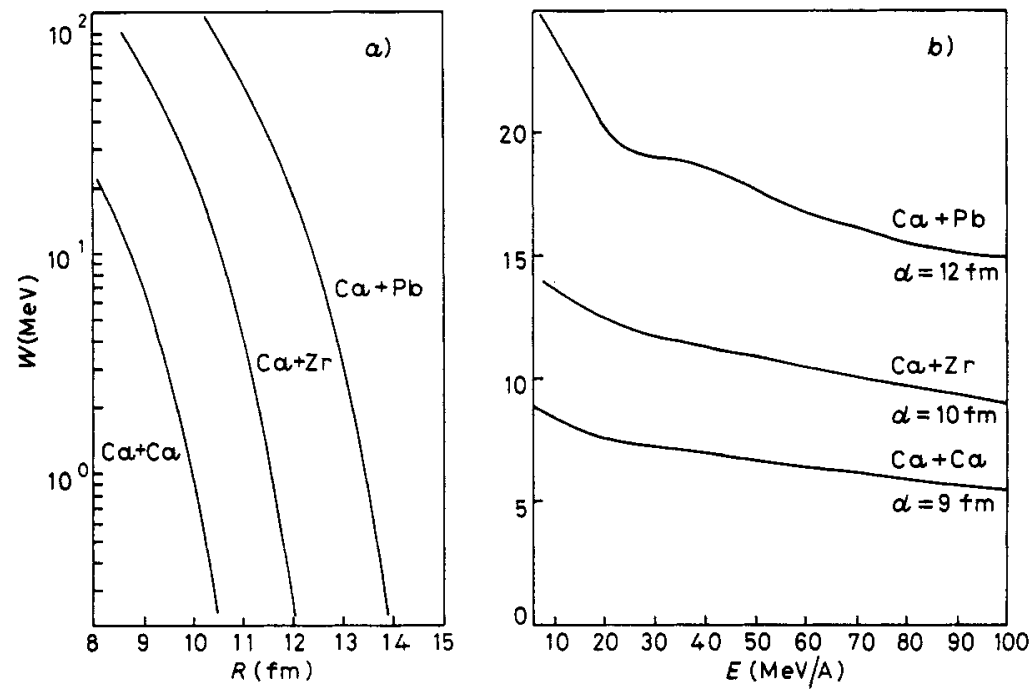

Fig. 2. - Imaginary potential associated through eq. (7) to the inelastic excitation in the three reactions $\mathrm{Ca}+\mathrm{Ca}, \mathrm{Ca}+\mathrm{Zr}, \mathrm{Ca}+\mathrm{Pb}:$ a) plotted as a function of the relative distance $R$ for a fixed incident energy $E_{\text {lab }}=33 \mathrm{MeV}$ per nucleon; $b$ ) plotted as a function of the incident energy for grazing relative distances, namely $R=12 \mathrm{fm}$ for $\mathrm{Ca}+\mathrm{Pb}, R=10 \mathrm{fm}$ for $\mathrm{Ca}+\mathrm{Zr}, R=9 \mathrm{fm}$ for $\mathrm{Ca}+\mathrm{Ca}$.

energy for radii close to the distance of closest approach associated with the grazing trajectory. From these figures one can see that the imaginary potential is strong and decreases slowly with incident energy in the 10 to $100 \mathrm{MeV}$ per nucleon range. This decrease can be partly related, through eq. (7), to the decrease of the reaction time. The present calculations were not performed below $10 \mathrm{MeV}$ per nucleon but the general pattern is a decrease of the inelastic imaginary potential when one approaches the barrier [7].

To get a deeper insight into both the shape and the incident energy evolution of the imaginary potential we have analysed separately the contributions of the target and of the projectile and we have furthermore decomposed the respective contributions into two parts:

i) one part which is due to low-lying excitations $\left(E^{*}<B\right)$;

ii) the other which comes from the excitation of high-energy states such as giant resonances $\left(E^{*}>B\right)$.

The limit $B$ between high-lying and low-lying states was chosen to be the particle emission threshold. The results of this analysis, plotted in percentage of the total absorption potential $W_{\mathrm{T}}$, are displayed in fig. 3 for the three reactions studied. In fig. $3 a$ ) the radial dependence of the various $W / W_{\mathrm{T}}$ is presented for the $33 \mathrm{MeV} /$ nucleon reactions, while their incident energy evolution is illustrated in fig. $3 b$ ) for grazing relative distances. Several remarkable features can be observed in this figure. First of all in asymmetric systems the projectile (the lighter nucleus) is much more excited than the target. This is in agreement with previous calculations $[6,8]$.

The important result is that giant-resonance excitations dominate the surface and longrange properties of the imaginary potential. For example, in the $\mathrm{Ca}+\mathrm{Ca}$ case, the slope of the exponential decrease of $W$ is found to be $0.45 \mathrm{fm}$ for the low-lying state component and 0.54 for the giant-resonance contribution. This difference does not arise from form factor shapes which are very similar but is due to the dynamics of the reaction. Indeed, the value of $W$ at each $R$ is determined by considering a trajectory whose distance of closest approach is just $R$. The contribution of the various excited states to $W$ is determined by the Fourier 


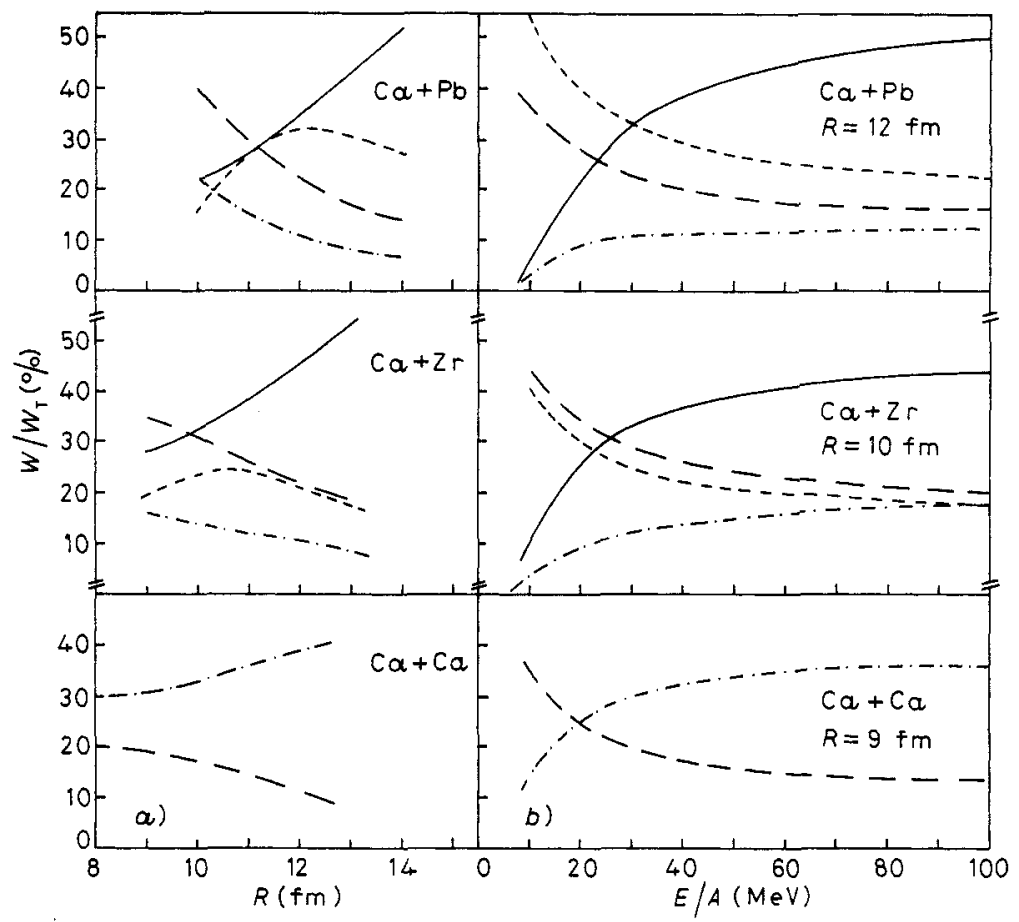

Fig. 3. - Decomposition of $W_{\mathrm{T}}$ in four parts. The contribution of low-lying state excitations in the projectile (small dash) and in the target (long dash) and of giant resonances in the projectile (solid line) and in the target (dotted dash). These four contributions are given in percentage of the total potential for the three studied reactions $\mathrm{Ca}+\mathrm{Ca}, \mathrm{Ca}+\mathrm{Zr}, \mathrm{Ca}+\mathrm{Pb}: a$ ) plotted as a function of the relative distance $R$ for a fixed incident energy $E_{\mathrm{lab}}=33 \mathrm{MeV}$ per nucleon; $b$ ) plotted as a function of the incident energy for grazing relative distance (see fig. 2b)).

transform of the form factors on each trajectory. For small distances this Fourier transform is concentrated at low frequencies, while for more distant trajectories the frequency spectrum is broader. Indeed for small distances of closest approach the nuclear attraction modifies the trajectories and lengthens the time over which the excitations occur. Thus the absorption due to high-frequency modes (like giant resonances) relatively to that induced by low-lying states is more important at large distances.

These results contradict the validity of using simple trajectories (straight lines [13] or pure Coulomb trajectories [6]) for the calculation of $\mathcal{I}$ (or $W$ ). Within such approximations the number of phonon can be factorized into

$$
\bar{n}_{i}=|\langle i|V(D)| 0\rangle|^{2} S_{\lambda_{i}}\left(\omega_{i 0}, D\right),
$$

where $S$ is a cut-off factor depending mainly on the multipolarity $\lambda$ and on the excitation energy $\omega$ and has a very small $D$-dependence. Therefore, the relative excitation of low-lying $v s$. high-lying states will not exhibit the strong radial dependence observed in fig. $3 a$ ). It must be noted that these conclusions are independent of the procedure chosen for the extraction of $W$, since the same radial dependence is already observed at the level of the mean numbers of excited phonons and thus of $|S|^{2}$.

Finally fig. $3 b$ ) shows that the dominance of giant-resonance excitation is stronger at high energy. In fact two regimes can be defined for the inelastic imaginary potential: a lowenergy regime which is characterized by low-lying state excitation and a high-energy regime 
where giant resonances are the most important degrees of freedom. For example, the transition between these two regimes appears as a small plateau around $30 \mathrm{MeV} / \mathrm{A}$ in the energy evolution of $W$ in the case of the $\mathrm{Ca}+\mathrm{Pb}$ reaction (fig. $2 b$ )).

In conclusion we have shown that, at intermediate incident energies $(E>20 \mathrm{MeV}$ per nucleon), the surface properties of the imaginary potential are related to the excitation of giant resonances. This excitation is stronger in the lighter nucleus. The resulting potentials have thus a longer range than those calculated including only low-lying states. When describing the surface interaction properties, for example in peripheral reactions, giant resonances appear as one of the most important degrees of freedom.

\section{REFERENCES}

[1] Buenerd M. et al., Phys. Rev. Lett., 40 (1978) 1482; Doll P. et al., Phys. Rev. Lett., 42 (1979) 366; Bertrand F. E., Nucl. Phys. A, 354 (1981) 129c; van Der Woude A., Prog. Nucl. Part. Phys., 18 (1987) 217.

[2] Barrette J. et al., Phys. Lett. B, 209 (1988) 182; SuomiJärvi T. et al., to appear in Nucl. Phys.

[3] Chomaz Ph. et al., Z. Phys. A, 319 (1984) 167; Catara F. and Lombardo U., Nucl. Phys. A, 455 (1986) 158; Catara F., Chomaz Ph. and VitTuri A., Nucl. Phys. A, 471 (1987) 661; Chomaz Ph., Van Giai N. and Vautherin D., Nucl. Phys. A, 476 (1988) 125.

[4] Blumenfeld Y. and Chomaz Ph., Phys. Rev. C, 38 (1988) 2157.

[5] Frascaria N. et al., Nucl. Phys. A, 474 (1987) 253; Frascaria N., Nucl. Phys. A, 482 (1988) $245 \mathrm{c}$.

[6] Broglia R. A., Pollarolo G. and Winther Aa., Nucl. Phys. A, 361 (1981) 307; Pollarolo G., Broglia R. A. and Winther Aa., Nucl. Phys. A, 406 (1983) 369; Dasso C. H., Landowne S., Pollarolo G. and Winther Aa., Nucl. Phys. A, 459 (1986) 134.

[7] Catara F. and Chomaz Ph., Nucl. Phys. A, 482 (1988) 271c; Andrés M. V., Catara F., Chomaz Ph. and LANZA E. G., Proceedings of the XXVI International Winter Meeting on Nuclear Physics, Bormio Italy 1988, p. 578; ANDRÉs M. V., CatARA F., Chomaz PH. and Lanza E. G., Phys. Rev. C, 39 (1989) 99.

[8] Vinh MaU N., Nucl. Phys. A, 457 (1986) 413; 470 (1987) 406.

[9] Satchler G. R., Phys. Lett. B, 55 (1975) 167; 59 (1975) 121.

[10] Pollarolo G., Broglia R. A. and Dasso C. H., Nucl. Phys. A, 451 (1986) 122.

[11] Chomaz Ph. and Vautherin D., Phys. Lett. B, 177 (1986) 9.

[12] Dechargé J., Gogny D., Grammaticos B. and Sips L., Phys. Rev. Lett., 49 (1982) 982; Dechargé J. and Sips L., Nucl. Phys. A, 407 (1983) 1.

[13] Chomaz Ph. and Vautherin D., Phys. Lett. B, 139 (1984) 244. 